\title{
Trends: cyborg games
}

\author{
Ian Yeoman and Una McMahon-Beattie
}

Dr lan Yeoman is an Associate Professor of Tourism Futures,

School of Management at

Victoria University of

Wellington, Wellington,

New Zealand.

Professor Una

McMahon-Beattie is the

Head of the Department of

Hospitality and Tourism

Management at Ulster

Business School, Ulster

University, Belfast, UK. lan Yeoman and

Una McMahon-Beattie.

Published in the Journal of Tourism

Futures. This article is published

under the Creative Commons

Attribution (CC BY 4.0) licence.

Anyone may reproduce, distribute,

translate and create derivative

works of this article (for both

commercial and non-commercial

purposes), subject to full attribution

to the original publication and

authors. The full terms of this

licence may be seen at:

http://creativecommons.org/

licences/by/4.0/legalcode

\begin{abstract}
Purpose - Wearable technologies are a near future concept and cyborgs are in fact reality. The authors' proposition is how cyborgisation could and will occur. The paper aims to discuss this issue.

Design/methodology/approach - The approach used by this paper is a general review.

Findings - The authors explain how the line between humans and technology is becoming more and more blurred as this trends paper explores the concepts of singularity and cyborgs as a future state highlighting the world's first cyborg games.
\end{abstract}

Originality/value - The paper contributes to our understanding that science fiction is fiction to some but reality to others depending on a person's cognition and insight.

Keywords Future, Sport, Science fiction, Events, Wearable technology, Singularity

Paper type General review

\section{Introduction: wearable technologies of the near future}

Computers have been part of everyday life since many of us can remember but as we move towards the point of singularity, where the processing power of the computer equals or surpasses that of the human brain, science fiction will become a reality. Simply put, the journey to singularity draws on the realms of science fiction, however, science fiction is often based on current developments in the best universities and technology companies of the world.

From a consumer perspective, the computers now have the ability to interact with us in a meaningful way. They talk to us in Russian, Mandarin, German or English in a natural way. For example, we all at some stage will have used Google translator. Interfaces are inconspicuous and natural and have transcended the keyboard and mouse. Now, new control mechanisms promise to comprehend even the subtlest nuances of our body language with dexterity we could only expect from another human being. Technology now operates in a sphere of integration and co-operation to the point that the "human" and "computer" are blended into one as in the cyborg. Brainwave command has already made its way into toys and fighter jets and engineers are today designing on-body control mechanisms and cameras that can successfully read emotions (Yeoman, 2012). These are known as wearable technologies and are devices made more useful by augmenting the capabilities of body. The most well known example here has been Google Glass (google.com/glass/start/) which uses an optical head mounted display like a hands free smart phone that can communicate with the internet, overlay augmented content and take photographs.

Computers are finding themselves empathetic, gaining awareness of our huge range of physical signals. They will soon be translating our body language with greater success than we do (Future Foundation, 2014). The idea of issuing an instruction will seem archaically cumbersome and human demand will simply equate to machine command. For example, BioBeats (biobeats.com) generates music from one or more heartbeats that builds content from emotion whereas the Emotiv (emotive.com) is a neuroheadset that uses a set of sensors to tune into 
electronic signals that allows gamers to use thought power across a whole range of online and video games. Nanobioelectronics (nanobiosensors.org) is a project that is exploring the commercial possibilities of temporary tattoos that can analyse the chemical constituents of sweat and thus help users monitor, in real-time, their fitness level, physical performance, electrolyte balance and hydration level which are invaluable metrics for athletes and fitness professionals. The non-invasive system could replace, it is hoped, alternative and invasive means of gathering such information. Whereas Li et al. (2014) have created a "wearable oral sensory system". This is a sensor that could be embedded into a tooth and monitor various in-mouth activities relating, for example, to diet and speech.

\section{Singularity and cyborgs}

Such is the pace of discovery and progress in technology we will see more innovation in the next ten years compared to the last 150 years (Yeoman, 2012). The advent of human-level artificial intelligence (Al) is on the horizon. The development of cyborg style professional athletes is the field of futurist Ray Kurzweil (2005) who proposes that exponential improvement described by Moore's Law will ultimately lead to a technological singularity. This is a period where progress in technology occurs almost instantly based upon the assumption that new types of technology will emerge such as optical or quantum computers. Kurzweil proposition about singularity envisages a point where the functionality of the human brain is quantifiable and matched by technology. Singularity means being on the verge of robots as humans. This is the point in the future where the singularity cyborg emerges as a professional athlete, a half-human, half-robot which is something akin to Lee Majors and The Six Million Dollar Man (Pilato, 2007).

A cyborg is a cybernetic organism coined by Clynes and Klines (1960) who used it an article about the advantages of self-regulating human-machines in Outer Space. The role of cyborgs today is associated with the most basic metaphysical, physical attachments, humanity and technology. For example, a human fitted with a heart pacemaker or an insulin pump might be considered a cyborg, since these mechanical parts enhance the body's natural mechanisms through synthetic feedback mechanisms. In modern medicine, the term cyborg is used to refer to a man or woman with a bionic or robotic implant.

\section{The cyborg games: a competition for augmented humans}

The use of cyborgs in sport is familiar to us all. Consumers will be familiar with the Olympian athlete Oscar Pistorious, the sprinter, who uses a pair of carbon fibre prosthetic limbs. The Olympic Games is a competition for the fittest and most talented able bodied, whilst the Paralympic Games is a competition for those with physical and intellectual disabilities. Pistorious, however, with the help of science, was able to compete in the Olympics. Indeed with the advancement of science and technology the concept of singularity is therefore becoming a reality and cyborgs are now more commonplace in a blended reality society of humanity. Prosthetics limbs are transitioning from passive systems to active ones, capable of sensing a user's intent (through nerve or brain interfaces) and use motors and actuators to more effectively replace a real limb. The goal now is to be able to provide capabilities similar to that of a human limb. For that reason the Swiss National Competence Center of Research in Robotics are organized the first cyborg games. Cybathlon (2014) states:

The Cybathlon is a championship for racing pilots with disabilities (i.e. parathletes) who are using advanced assistive devices including robotic technologies. The competitions are comprised by different disciplines that apply the most modern powered knee prostheses, wearable arm prostheses, powered exoskeletons, powered wheelchairs, electrically stimulated muscles and novel brain-computer interfaces. The assistive devices can include commercially available products provided by companies, but also prototypes developed by research labs. There will be two medals for each competition, one for the pilot, who is driving the device, and one for the provider of the device.

The games will include a powered arm prosthetics competition in which pilots with forearm or upper arm amputations will be equipped with actuated exoprosthetic devices and will 
have to successfully complete two hand-arm task courses as quickly as possible. The Brain-Computer interface race pilots control an avatar in a racing game played on computers whereas the powered Exoskeleton Race pilots will complete with thoracic or lumbar spinal cord injuries and will be equipped with actuated exosketal devices. Other races include electrical simulation bikes, powered leg prosthetics and powered wheelchairs. These examples highlight how cyborgs and wearable technologies will become commonplace in our society (see Figures 1 and 2).

\section{Figure 1 Powered leg prosthetics}

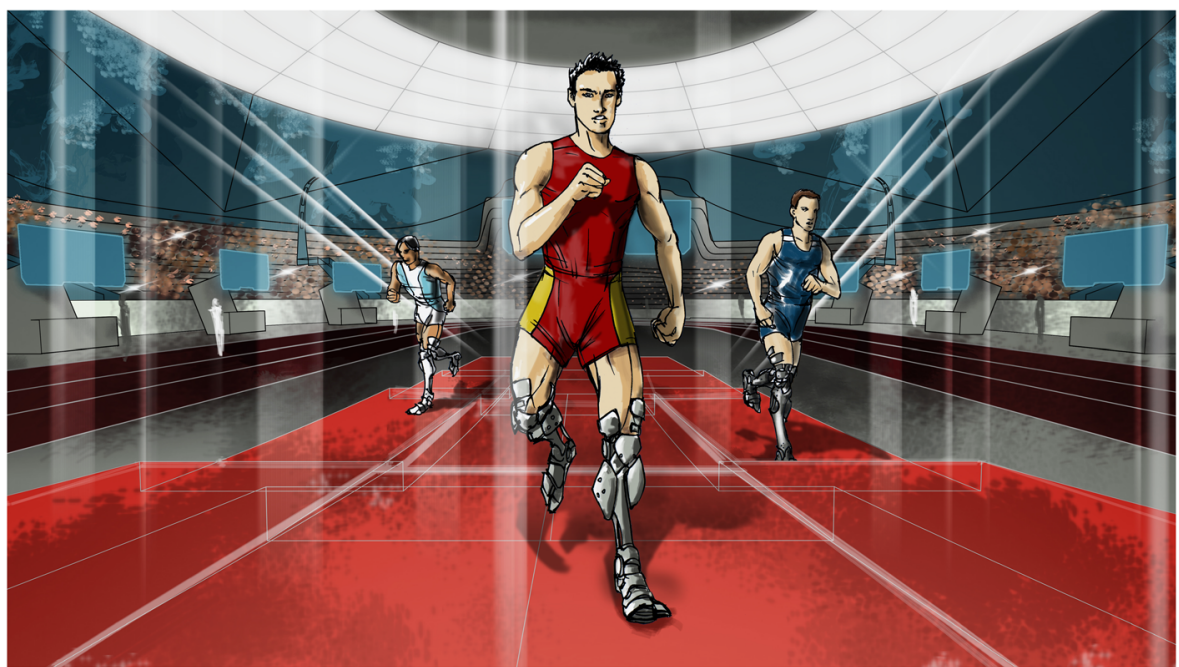

Source: Courtesy of cybathlon.ethz.ch and D'Arc. Studio Associates Architects

\section{Figure 2 Brain-computer interface race}

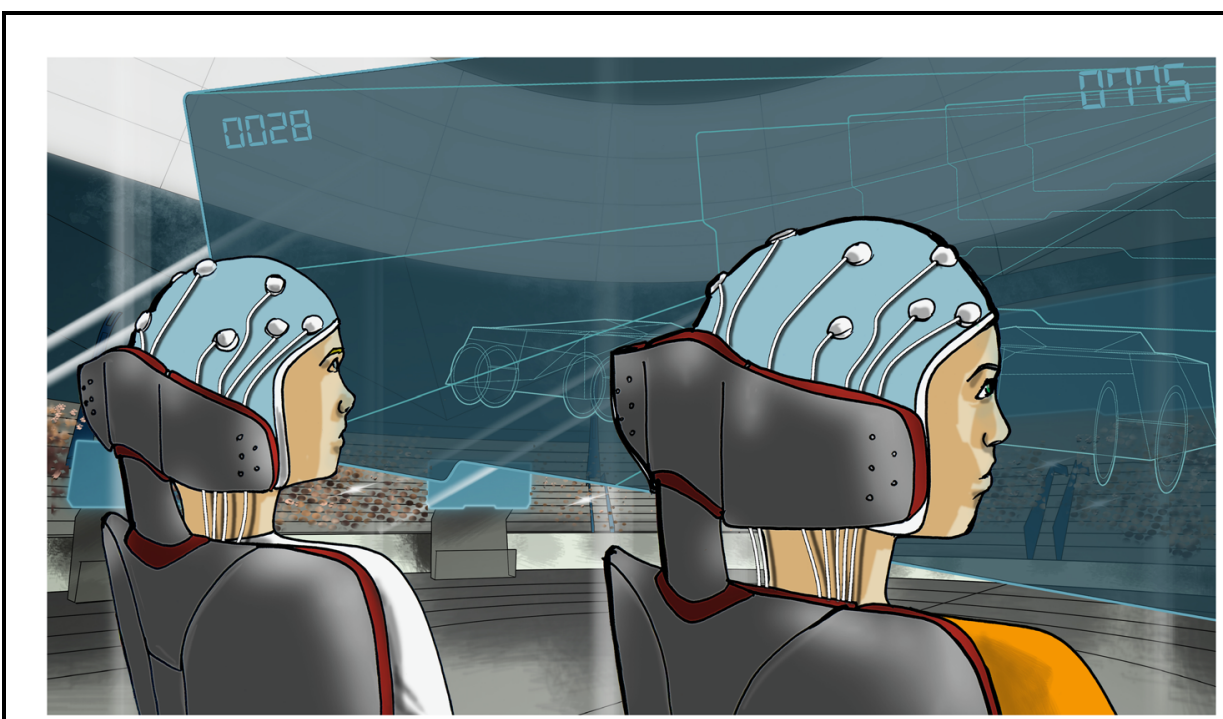

Source: Courtesy of cybathlon.ethz.ch and D'Arc. Studio Associates Architects 


\section{References}

Clynes, M. and Klines, S. (1960), "Cyborgs and space”, available at: http://cyberneticzoo.com/wp-content/ uploads/2012/01/cyborgs-Astronautics-sep1960.pdf (accessed 30 March 2014).

Cybathlon (2014), "CYBATHLON 2016: the championship for robot-assisted parathletes", available at: www.cybathlon.ethz.ch/ (accessed 30 March 2014).

Future Foundation (2014), "Catwalk computing", available at: http://nvision.futurefoundation.net/ (accessed 30 March 2014).

Kurzweil, R. (2005), The Singularity is Near, Penguin Books, New York, NY.

Li, C., Chen, Y., Chen, W., Huang, P. and Chu, H. (2014), "Senor embedded teeth for oral activity recognition", available at: http://mll.csie.ntu.edu.tw/papers/TeethProbelSWC.pdf (accessed 30 March 2014).

Pilato, H. (2007), Bionic Book Reconstructed, Bear Monar Media, Albany, NY.

Yeoman, I. (2012), 2050: Tomorrow's Tourism, Channelview, Bristol.

\section{Corresponding author}

Dr lan Yeoman can be contacted at: ian.yeoman@vuw.ac.nz 